\title{
A very narrow gyrosynchrotron spectrum during a solar flare
}

\author{
C. G. Giménez de Castro $^{1}$, J. E. R. Costa ${ }^{1,2}$, A. V. R. Silva ${ }^{1}$, P. J. A. Simões ${ }^{1,2}$, E. Correia ${ }^{1,2}$, and A. Magun ${ }^{3}$ \\ ${ }^{1}$ Centro de Rádio Astronomia e Astrofísica Mackenzie, R. da Consolação 896, 01302-907 São Paulo, SP, Brazil \\ e-mail: guigue@craam.mackenzie.br \\ 2 Instituto Nacional de Pesquisas Espaciais, São José dos Campos, Brazil \\ 3 Institute of Applied Physics, University of Bern, Bern, Switzerland
}

Received 28 October 2005 / Accepted 13 June 2006

\section{ABSTRACT}

\begin{abstract}
During the rising phase of the radio burst of August 30, 2002, at $\sim 1328$ UT a short pulse with a duration of approximately $4 \mathrm{~s}$ was observed. Here we present a multiwavelength analysis, including microwave and X-ray. Its background-subtracted radio spectrum ranges only from 2.5 to $12 \mathrm{GHz}$ with a maximum flux density of approximately 900 s.f.u. at $7 \mathrm{GHz}$ and a steep optically thin spectral index $\alpha \sim 8$. The hard X-ray pulse emission above the background in the range of 10-150 keV observed by RHESSI is coincident in time with the microwave observation. Hard X-ray images reveal very compact $\left(\sim 10^{\prime \prime}\right)$ footpoint sources. A distribution of accelerated electrons represented by a double power law, with $\delta_{E<250 \mathrm{keV}}=5.3$ and $\delta_{E} \geq 250 \mathrm{keV}=13$, was used to compute the expected gyrosynchrotron and thick target bremsstrahlung fluxes of a homogeneous source. We interpret the very steep electron index above the energy break to represent a high energy cutoff. With these parameters, our results reproduce the observations well. Nevertheless, they pose the still unanswered question about the mechanism that has slectively accelerated these electrons.
\end{abstract}

Key words. Sun: activity - Sun: flares - Sun: radio radiation

\section{Introduction}

Solar flares are produced by the release of magnetic energy stored in chromospheric/coronal loops. During the flare, the accelerated electrons emit gyrosynchrotron radiation at microwave frequencies along with hard X-rays (HXR) due to bremsstrahlung. These emissions come primarily from the Chromosphere/low Corona regions, and their observation provides the basic tool to diagnose the acceleration and transport of particles. Usually microwave emission is observed as a spectral broadband continuum in the range of approximately 1 to $50 \mathrm{GHz}$, and in some cases at even higher frequencies.

Narrowband short duration spikes that are superimposed onto the broadband emission are known to exist in the $1-5 \mathrm{GHz}$ range and are normally attributed to coherent plasma radiation (Benz 1986). Bruggmann et al. (1990) reported the existence of superimposed narrowband emission during solar flares in the range 6.2-8.2 GHz. They have observed three different types of structures: a) spikes with millisecond duration, b) frequency drifts with $\sim 1 \mathrm{~s}$ duration, and $\mathrm{c}$ ) in one case a patch structure with 15 s duration and a relative bandwidth of $20 \%$.

It is believed that the number density of the accelerated electrons can be well approximated by the power law $N(E)=K E^{-\delta}$ where the electron energy $E$ typically covers a range between $10 \mathrm{keV}$ and $1 \mathrm{MeV}$. The determination of the two energy cutoffs $E_{\min }$ and $E_{\max }$ provide insights into the acceleration mechanism. Holman (2003) has extensively analyzed the effects of low- and high-energy cutoffs on solar flare microwave and HXR spectra when gyrosynchrotron and bremsstrahlung radiation are considered. He has found that the density flux of the optically thin part of the microwave spectra falls faster than a power law and is better represented by a simple exponential, while the optically thick part of the microwave spectrum becomes flatter. In the
HXR domain the non-thermal bremsstrahlung spectra become steeper as the high energy cutoff decreases.

In this work we present a detailed analysis of a short burst during the impulsive phase of a X1.5 GOES class event that occurred near the limb in NOAA region 10095 (N15 E74) on August 30, 2002, at approximately 1328 UT. The main event will be studied elsewhere. The short impulsive peak lasts approximately 4 seconds and its microwave spectrum ranges from 2.5 to $12 \mathrm{GHz}$. It has some similar characteristics to the patch structure analyzed by Bruggmann et al. (1990), but with a spectral relative bandwidth $(>100 \%)$ much larger than theirs and a higher peak flux ( 1000 s.f.u.). We argue that the only explanation of such a spectrum is gyrosynchrotron emission from electrons whose density distribution severely decreases above $250 \mathrm{keV}$, implying some sort of small high energy cutoff like that studied by Holman (2003). We include HXR observations as well as total flux microwave data and polarization at $7 \mathrm{GHz}$ in this work. The next section describes the observations. Section 3 analyzes the microwave and HXR spectra obtained and, discusses the best solution. In Sect. 4, we present some final remarks.

\section{Observations}

The HXR data were obtained with the NASA Reuven Ramaty High Energy Solar Spectroscopic Imager (RHESSI), which provides imaging and spectral $\mathrm{HXR} / \gamma$-Ray observations with high spatial (up to $\sim 2^{\prime \prime}$ ) and spectral (up to $\sim 1 \mathrm{keV}$ ) resolution in the $\sim 3 \mathrm{keV}-17 \mathrm{MeV}$ energy range (Lin et al. 2002). Microwave radio data were gathered by the patrol telescopes of the Radio Solar Telescope Network (RSTN) at the frequencies of 2.695, 4.995 , and $8.8 \mathrm{GHz}$ with a time resolution of $1 \mathrm{~s}$. Left- and righthanded polarized observations at $7 \mathrm{GHz}$ were provided by the Solar Radiopolarimeter of the Radio Observatory of Itapetinga 


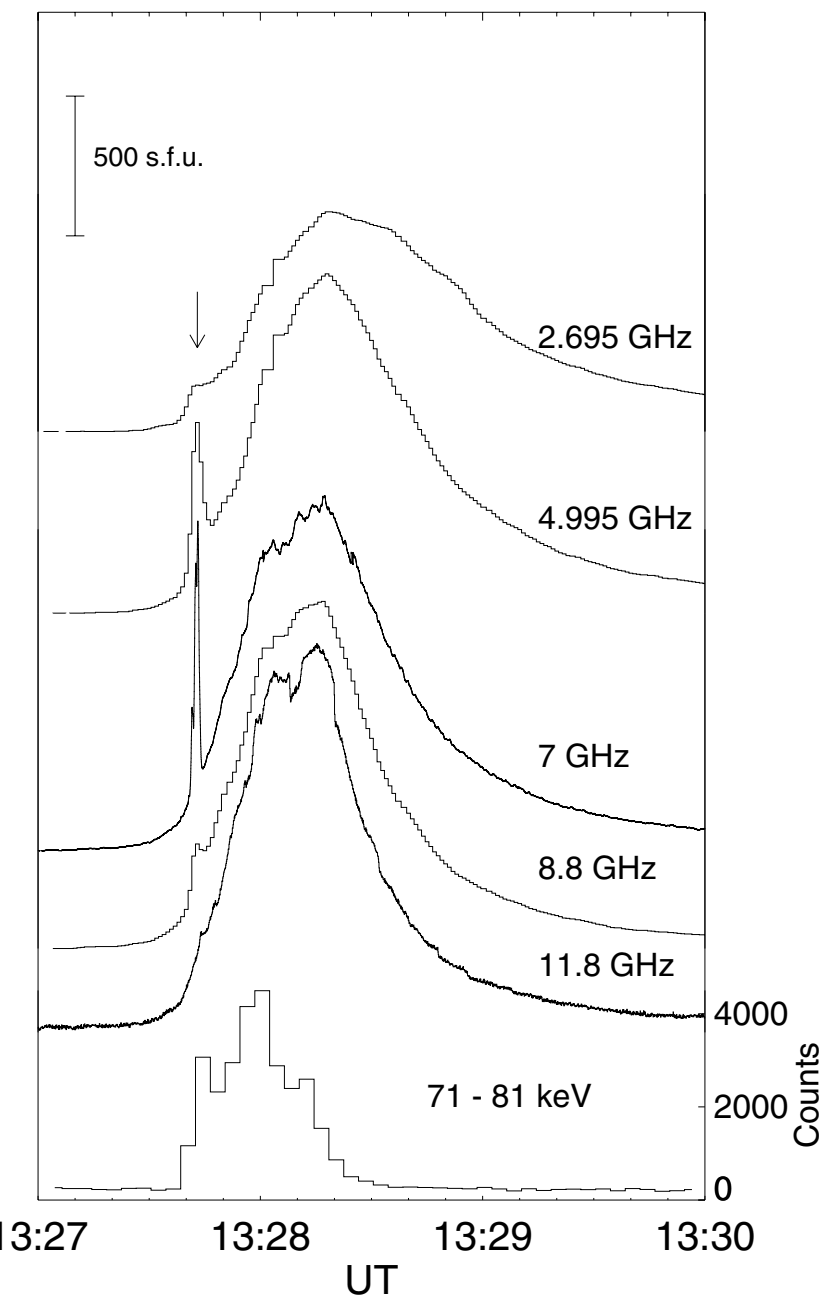

Fig. 1. Time evolution of the whole event at different microwave frequencies and in HXR at 71-81 keV. The arrow marks the small burst during the rising phase.

with 0.5 s.f.u. sensibility and $10 \mathrm{~ms}$ time resolution. We complete our multiwavelength observations with the total flux patrol telescopes of the University of Bern at the frequency of $11.8 \mathrm{GHz}$ with $100 \mathrm{~ms}$ temporal resolution and 10 s.f.u. sensitivity. All radio instruments have a total uncertainty around $10 \%$. As there is no overlap in frequency between different instruments that can be used as an intercalibration, we rely on each observatory's own calibration. In any case, the quoted uncertainty would not significantly change our results.

Figure 1 shows the time profiles at different wavelengths for the whole event. The short burst (or pulse) that is analyzed in this work is marked by an arrow. At $2.695 \mathrm{GHz}$ the pulse is a small enhancement over the gradually increasing flux of the flare. The flux density increases with frequency to a maximum near $7 \mathrm{GHz}$, then decreases at $8.8 \mathrm{GHz}$ and is almost not observed at $11.8 \mathrm{GHz}$. The corresponding HXR burst emission is significant up to $\sim 150 \mathrm{keV}$. The $71-81 \mathrm{keV}$ HXR time profile with a $4 \mathrm{~s}$ time resolution is shown in Fig. 1 as an example. Figure 2 shows the spectra just before the maximum of the pulse (1327:41 UT, asterisks in the figure), during maximum (1327:43 UT, triangles), and just after maximum (1327:45 UT, squares). The broadband component between 1 to $50 \mathrm{GHz}$ is seen in the three spectra with the flux increasing with time. During the peak time of the pulse, however, a different component between 2 and $12 \mathrm{GHz}$ is seen

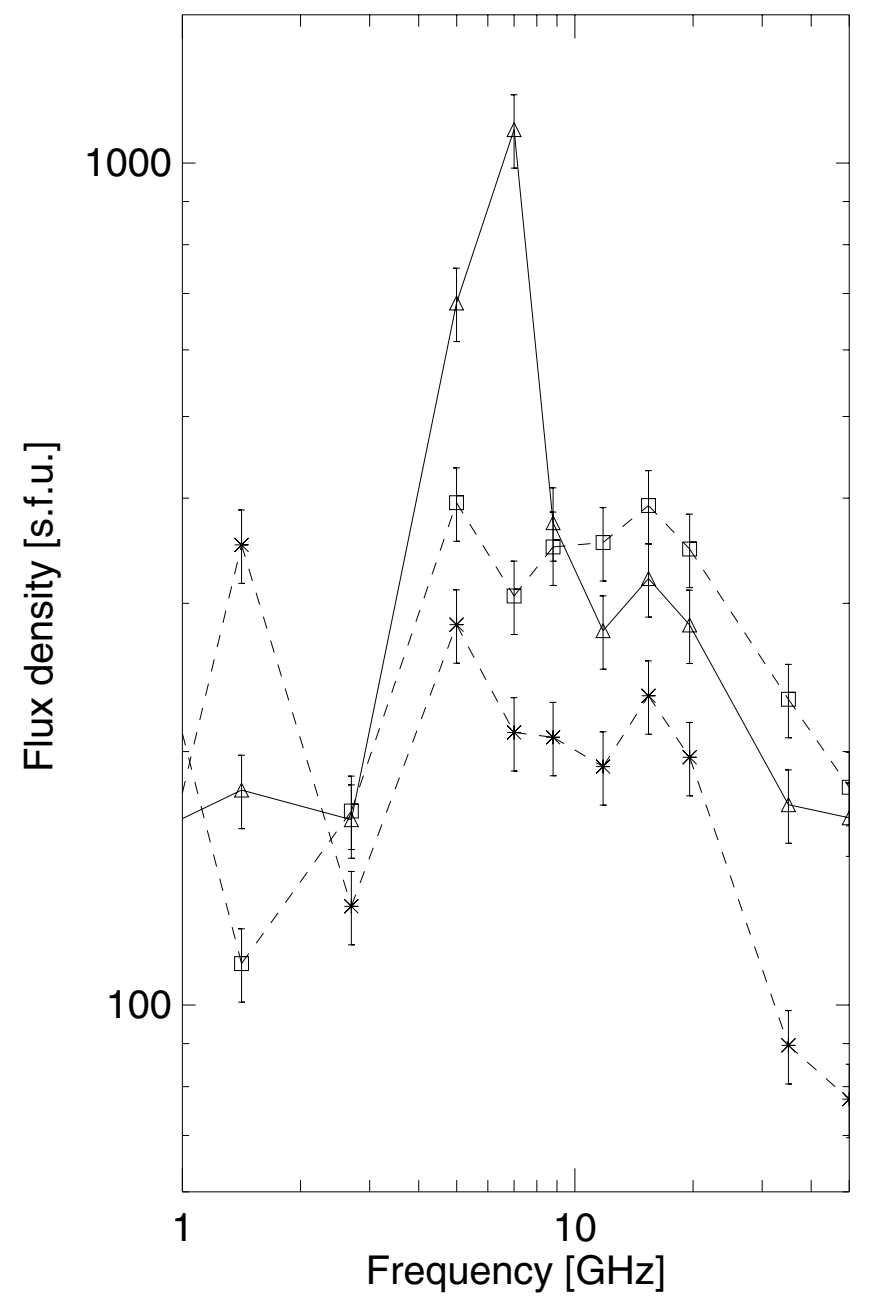

Fig. 2. Microwave spectra between 1-50 GHz at three different times. Asterisks represent flux density at 1327:41 UT, triangles at 1327:43 UT (peak time of the pulse), and squares at 1327:45 UT.

with a peak at $7 \mathrm{GHz}$. The analysis of this different spectral component is the aim of the present work.

During the pulse we removed the background due to the gradual increase of the flare emission. Part of the spectrum of the pulse emission is optically thick and is embedded in the optically thick regime of the flare. Since there are no radio images, it is not possible to know whether the emitting sources of the pulse and the flare overlap or not, and thus we cannot completely justify this procedure. Nevertheless the background subtraction does not change the main results of our work, as we will show later.

Figure 3 shows the resulting time profiles at 4.995, 7.0, and $8.8 \mathrm{GHz}$. The frequencies have different time resolutions, namely, $1 \mathrm{~s}$ at 4.995 and $8.8 \mathrm{GHz}$ and $20 \mathrm{~ms}$ at $7.0 \mathrm{GHz}$. The HXR background subtracted burst flux in the $64-67 \mathrm{keV}$ band is also presented in the same figure. As deduced from the $7 \mathrm{GHz}$ data, the peak time is $t_{\mathrm{p}}=1327: 43.12 \mathrm{UT}( \pm 0.02 \mathrm{~s})$. At this frequency the pulse has three peaks, each with a duration of approximately $1 \mathrm{~s}$. At the other two microwave frequencies the time resolution does not allow us to resolve these structures. The starting times for the three frequencies coincide within the RSTN resolution time (1 s), however, the duration appears to be shorter at $7 \mathrm{GHz}$. The peak time of the HXR emission is 1327:44 UT ( $\pm 2 \mathrm{~s})$, in agreement with that at microwaves. The bottom plot of Fig. 3 shows the degree of circular polarization 


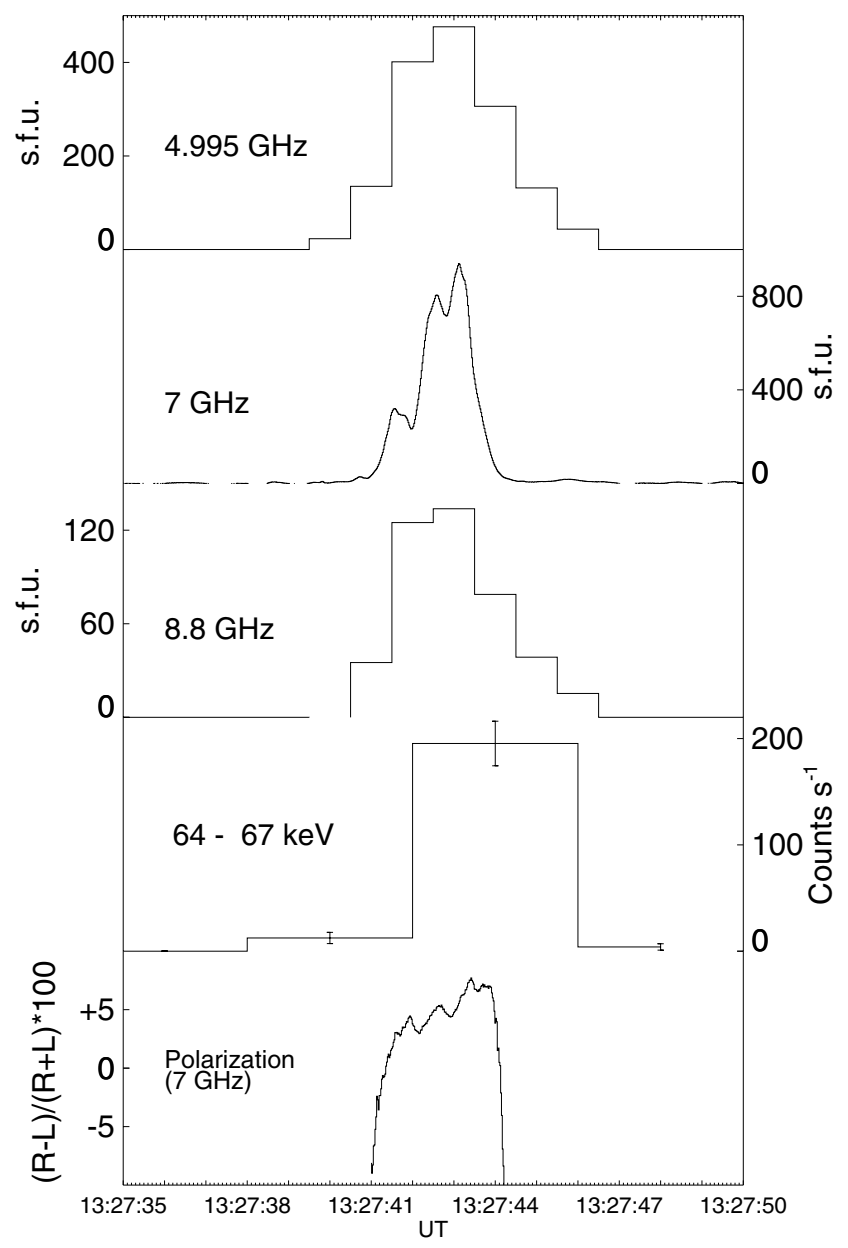

Fig. 3. Time evolution of the background-subtracted flux densities at microwaves and 64-67 keV HXR counts. The error bars in the HXR data represent three times the standard deviation of a Poisson distribution. The bottom plot represents the degree of circular polarization of the $7 \mathrm{GHz}$ signal.

of the background-subtracted signal at $7 \mathrm{GHz}$ between 1347:41 and 1347:45 UT, when the flux density is significant. It varies between -10 and $5 \%$ and becomes maximum at the peak time with a statistical error of $\pm 0.9 \%$.

The extreme-ultraviolet (EUV) images from TRACE show a complex system of magnetic arches (Fig. 4). A compact bright spot coincides spatially with the 50-100 keV emission (Fig. 4) with its peak time delayed by approximately $6 \mathrm{~min}$. The HXR emission comes from a compact source. At the $50 \%$ flux level we see two spots: the eastern one has an elongated shape oriented in the East-West direction with a projected dimension of $8^{\prime \prime} \times 3^{\prime \prime}$ that could be further subdivided into two sources. The western source is round with a $\sim 3^{\prime \prime}$ diameter. When the $10 \%$ flux level is considered, the source dimension increases to a square with a $10^{\prime \prime}$ side length.

For spectral analysis we have integrated all microwave data over $1 \mathrm{~s}$. Figure 5 shows the background-subtracted microwave spectrum during the peak time. In the same figure we also plotted the non-subtracted signal for the frequencies of 2.695 and $4.995 \mathrm{GHz}$, the two optically thick frequencies of the pulse. Because of the integration process, the flux at $7 \mathrm{GHz}$ is reduced from 940 to 860 s.f.u. The error bars represent the statistical flux errors after subtraction of the background.

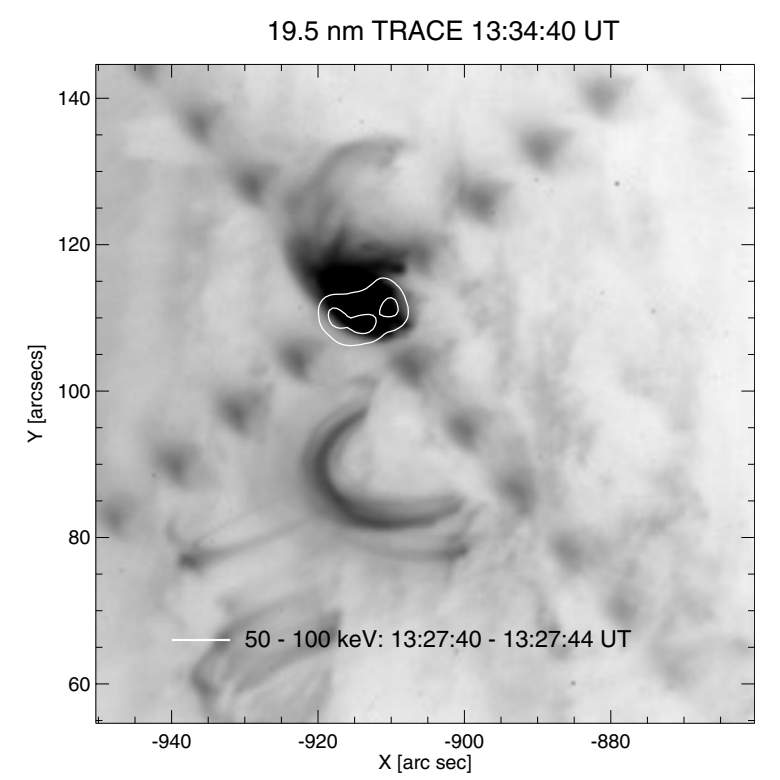

Fig. 4. A negative TRACE image $(\lambda=19.5 \mathrm{~nm})$ obtained during the maximum of the EUV emission (1334:40 UT). White contours represent a $50-100 \mathrm{keV}$ image obtained by RHESSI during the interval of the pulse (1327:40-1327:44 UT). The brightest EUV and HXR emission coincide in a compact region of $\sim 15^{\prime \prime} \times 10^{\prime \prime}$. A diffraction pattern of the instrument is also seen. Top is North, right is West.

\section{Discussion}

\subsection{Origin of the radiation}

It is clear from the time profiles and spectra that this pulse requires extreme conditions in order to be explained by current models. The first idea that comes to mind is that of coherent plasma or maser emission. Staehli et al. (1987) have found evidence of coherent harmonic microwave radiation during solar flares in patrol observations with the Bern telescopes with almost the same spectral resolution. Harmonics were observed at two different frequencies with a ratio of approximately 1:2 and with durations of seconds. In our case we observe the pulse between 2.695 and $11.8 \mathrm{GHz}$ that corresponds to a $9 \mathrm{GHz}$ bandwidth at a center frequency of approximately $7 \mathrm{GHz}$. This excludes the possibility of microwave coherent emission. Moreover, because of the low polarization (Fig. 3), any type of maser emission can be rejected.

The spectral index of the optically thin part $\alpha_{\text {thin }}=8.0$ yields an electron index $\delta=10.2$ using the Dulk (1985) approximations. If we consider a single power law electron distribution, such an index will concentrate all the electrons in the low energy cutoff (e.g., $10 \mathrm{keV}$ ), and a huge number of electrons is needed to achieve the observed high HXR and microwave fluxes. Bruggmann et al. (1990) showed that a source of monoenergetic electrons of $11 \mathrm{keV}$ in a magnetic field of $290 \mathrm{G}$ produces gyrosynchrotron emission with a peak at $7 \mathrm{GHz}$, with a bandwidth of $3 \mathrm{GHz}$ that is narrower than that of our pulse. Moreover, to accomplish the observed flux density, an unrealistic source size and total number of electrons are needed.

Instead of monoenergetic electrons or a single power law, we consider a double power law distribution steep enough to reduce the high frequency emission. To derive physical parameters of the emitting sources, we have simultaneously fitted both the HXR and microwave spectra using homogeneous models of thick target bremsstrahlung and gyrosynchrotron emission, respectively. The bremsstrahlung was calculated by means of the 


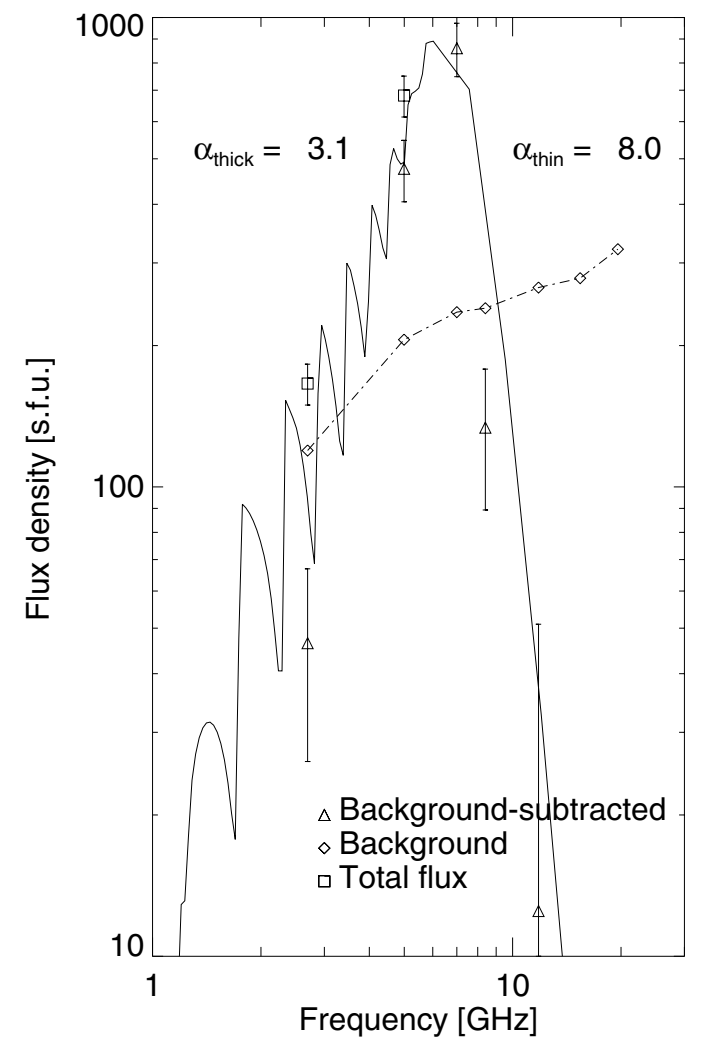

Fig. 5. Background-subtracted microwave spectrum during the peak time (triangles), where $\alpha_{\text {thick }}$ and $\alpha_{\text {thin }}$ are the values of the spectral indices, respectively. Diamonds (dotted curve) represent the background (gradual) spectrum. Squares are the non-subtracted flux for the optically thick part of the pulse. The solid curve is the modeled flux of a homogeneous source (see text).

numerical code provided by Holman (2003). The RHESSI image was used to obtain the HXR source area $A_{\mathrm{X}}=3.3 \times 10^{17} \mathrm{~cm}^{2}$, considering the flux at $10 \%$ level. We have adapted the Ramaty et al. (1994) gyrosynchrotron code to accept a double power-law electron distribution. We note that for bremsstrahlung computations the electron flux is required, whereas for gyrosynchrotron calculations the electron instant number is needed. This creates a difference in the electron index. We call $\delta_{\mathrm{X}}$, the index inferred from a non-thermal thick target bremsstrahlung emission model and $\delta_{\mathrm{R}} \equiv \delta$ the index inferred from a gyrosynchrotron emission model. In this work we have considered $\delta_{\mathrm{X}}=\delta-0.5$ (see, e.g., Trottet et al. 1998). The best fit to the spectra was sought using an iterative process where only $A_{\mathrm{X}}$ and the high energy cutoff of the power-law distribution were fixed. In a first run we used a very broad range of parameter values, with a coarse variation and obtained rough estimates of the magnetic field, electron indices, break energy, and electron density. The final run consisted of around two million solutions obtained from a restricted set of parameters and fine variations that were compared with the observed spectra; the standard deviation was used as a quality indicator. The range of the parameters of the final iteration is shown in Table 1 along with those values that best fitted the observed pulse spectrum. It should be emphasized that the set of acceptable solutions has a very limited interval for the magnetic field and gyrosynchrotron source size $\left(A_{\mathrm{s}}\right)$, giving little space for other values. We note that the optically thick part of the fitting is indifferent to the background subtraction process.

The fitted radio and HXR spectra are shown in Figs. 5 and 6, respectively. The fit of the HXR spectrum is very good above
Table 1. Derived parameters from microwave spectral fits.

\begin{tabular}{l|rrrl}
\hline \hline Param. & Min. & Max. & Fit & Units \\
\hline$N_{\mathrm{el}}^{a}$ & $10^{6.7}$ & $10^{8.7}$ & $9.510^{7}$ & $\mathrm{~cm}^{-3}$ \\
$E_{\min }$ & 20 & 35 & 35 & $\mathrm{keV}$ \\
$E_{\mathrm{brk}}$ & 120 & 300 & 250 & $\mathrm{keV}$ \\
$E_{\max }$ & & & 800 & $\mathrm{keV}$ \\
$\delta_{E<E_{\text {brk }}}^{b}$ & 4.2 & 5.8 & 5.3 & \\
$\delta_{E>E_{\text {brk }}}^{c}$ & 9.0 & 15.0 & 13.0 & \\
$B_{\operatorname{mag}}$ & 180 & 400 & 215 & $\mathrm{G}$ \\
$\alpha_{\mathrm{R}}^{d}$ & 0.5 & 3.5 & 1.2 & \\
$\cos (\theta)^{e}$ & 0.05 & 0.3 & 0.1 & \\
$L_{\mathrm{s}}^{f}$ & $10^{8.7}$ & $10^{9.3}$ & $10^{9}$ & $\mathrm{~cm}$ \\
$S_{\mathrm{s}}^{g}$ & 30 & 60 & 40 & $\prime \prime$ \\
$A_{\mathrm{s}}^{h}$ & & & $6 \times 10^{18}$ & $\mathrm{~cm}^{2}$ \\
$A_{\mathrm{X}}^{i}$ & & & $3.3 \times 10^{17}$ & $\mathrm{~cm}^{2}$ \\
$N_{\text {tot }}^{j}$ & & & $6 \times 10^{35}$ & \\
\hline
\end{tabular}

${ }^{a}$ Number density of accelerated $e-$ with $E>E_{\min } ;{ }^{b}$ electron index below break energy; ${ }^{c}$ electron index above break energy; ${ }^{d} 1.5 v_{\mathrm{B}} / v_{\mathrm{p}}$ (Razin's factor); ${ }^{e}$ cosine of the view angle; ${ }^{f}$ source depth; ${ }^{g}$ source diameter; ${ }^{h}$ emitting area; ${ }^{i}$ HXR emitting area; ${ }^{j}$ total number of accelerated electrons.

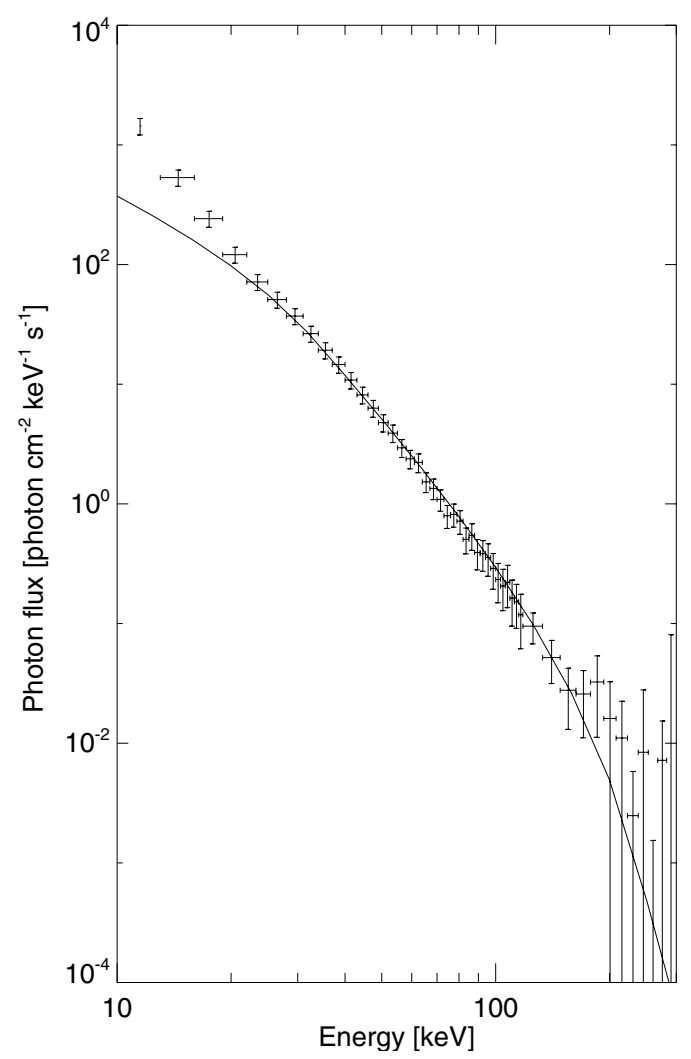

Fig. 6. Background-subtracted HXR spectrum at the peak time. Error bars are equal to $3 \sigma$ of the photon flux distribution. The solid curve represents the modeled flux (see text).

$35 \mathrm{keV}$, while below a different component, probably of thermal origin, is seen. Due to the increased uncertainty of the data above $150 \mathrm{keV}$, we have not attempted to fit it. The microwave fit is good at $4.995,7 \mathrm{GHz}$ and $11.8 \mathrm{GHz}$, but only marginal at 2.695 and $8.8 \mathrm{GHz}$. The obtained viewing angle, $84^{\circ}$, is consistent with an arch close to the solar limb that is perpendicular to the solar surface. 
We note that we could have used a high energy cutoff at $250 \mathrm{keV}$, as Holman (2003) did, with no appreciable change in our results. We find our double power law less artificial. Double power laws have been extensively analyzed in the literature. Trottet et al. (1998), in an analysis of the June 11, 1990, event using the PHEBUS X-Ray $/ \gamma$-ray telescope, found that the electron spectrum hardens above a few $\mathrm{MeV}$. On the other hand, Holman et al. (2003), using the RHESSI telescope, have shown a softening of the electron spectrum above a few keV during the July 23, 2002 , flare. In both cases, the change in the spectral index $\delta$ is around \pm 1 . In the present work, we propose a change in $\delta$ of around 8 , which is rather unusual.

\subsection{Comparison with models}

Holman (2003) found that a small high energy cutoff should steepen the optically thin part of the microwave spectrum resulting in an exponential shape instead of a power law. In addition to that a cutoff at $200 \mathrm{keV}$ produces a small flattening in the optically thick part of the spectrum (i.e., the spectral in$\operatorname{dex} \alpha$ is reduced from 3.5 to 2.9). Our observations agree with the steepening of the thin part of the spectrum. As we do not have enough frequency coverage, we cannot determine whether the shape is an exponential or a power law. The optically thick part of our microwave spectrum is not flattened, instead it shows some degree of medium suppression at lower frequencies (the so called Razin-Tsytovich effect, see, e.g., Dulk 1985), which has an steepening effect. In Table $1, \alpha_{\mathrm{R}}=1.2$, where $\alpha_{\mathrm{R}}=1.5 v_{\mathrm{B}} / v_{\mathrm{p}}$, with $v_{\mathrm{p}}$ and $v_{\mathrm{B}}$ as the plasma and gyrofrequencies respectively (Ramaty 1969).

On the other hand, the expected exponential HXR spectrum of a low high-energy cutoff (Holman 2003) is seen in our data from 35 up to $150 \mathrm{keV}$ (Fig. 6). Below $35 \mathrm{keV}$ there is probably a thermal component not included in the pure homogeneous thick target bremsstrahlung model analyzed by Holman.

\section{Final remarks}

This work presents a multiwavelength analysis of an unusual microwave burst, short in time, intense in flux, and with a narrow spectrum at maximum flux. This narrow spectrum is an indication of the absence of high energy electrons. The observations are best represented by gyrosynchrotron emission from electrons with a double power law: below a break energy $E_{\text {brk }}=250 \mathrm{keV}$ the electron index $\delta_{E<250 \mathrm{keV}}=5.3$, and above the break energy, $\delta_{E}>250 \mathrm{keV}=13$. Our conclusion is based on the simultaneous fitting of both the HXR and microwave emissions.

The $\delta_{E}>250 \mathrm{keV}=13$ index acts as an effective high energy cutoff. Therefore a population of electrons were accelerated to energies between a few tens to a few hundreds of $\mathrm{keV}$ only, which is rather unusual and raises the question about the acceleration mechanism.

Acknowledgements. The authors are grateful to Cristina H. Mandrini for her careful reading and fruitful discussions and to the RSTN, TRACE, and RHESSI teams for their data and helpful support. This work was partially supported by FAPESP, grant No.: 03/03406-6.

\section{References}

Benz, A. O. 1986, Sol. Phys., 104, 99

Bruggmann, G., Magun, A., Benz, A. O., \& Stehling, W. 1990, A\&A, 240, 506

Dulk, G. A. 1985, ARA\&A, 23, 169

Holman, G. D. 2003, ApJ, 586, 606

Holman, G. D., Sui, L., Schwartz, R. A., \& Emslie, A. G. 2003, ApJ, 595, L97

Lin, R. P., Dennis, B. R., Hurford, G. J., et al. 2002, Sol. Phys., 210, 3

Ramaty, R. 1969, ApJ, 158, 753

Ramaty, R., Schwartz, R. A., Enome, S., \& Nakajima, H. 1994, ApJ, 436, 941

Staehli, M., Magun, A., \& Schanda, E. 1987, Sol. Phys., 111, 181

Trottet, G., Vilmer, N., Barat, C., et al. 1998, A\&A, 334, 1099 